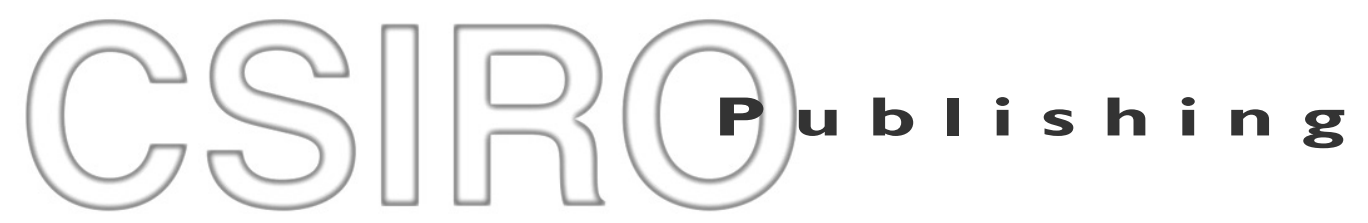

\title{
Publications of the Astronomical Society of Australia
}

Volume 18, 2001

(C) Astronomical Society of Australia 2001

An international journal of astronomy and astrophysics

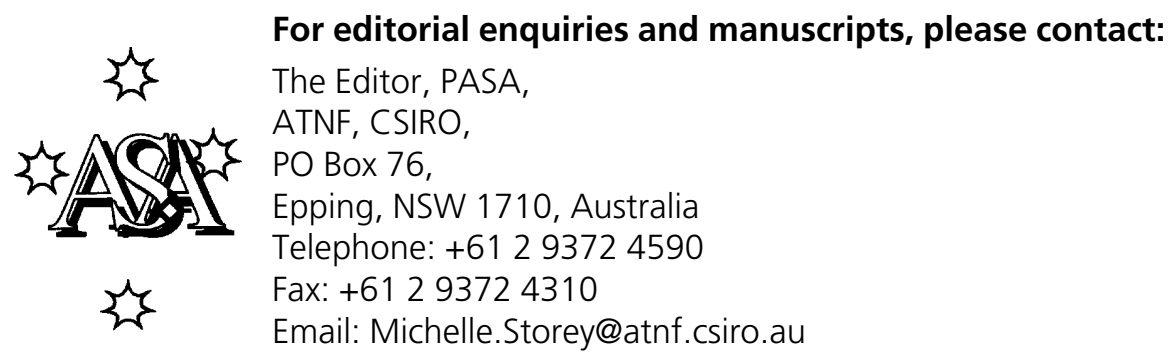

For general enquiries and subscriptions, please contact: CSIRO Publishing PO Box 1139 (150 Oxford St)

Collingwood, Vic. 3066, Australia

Telephone: +61 396627666

Fax: +61 396627555

Email: pasa@publish.csiro.au

C S I RO

PUBLISHING Published by CSIRO Publishing

for the Astronomical Society of Australia

www.publish.csiro.au/journals/pasa 


\title{
Lower Hybrid Drive in Solar Magnetic Reconnection Regions: Implications for Electron Acceleration and Solar Heating
}

\author{
Iver H. Cairns \\ School of Physics, University of Sydney, NSW 2006, Australia \\ cairns@physics.usyd.edu.au
}

Received 2001 April 24, accepted 2001 September 19

\begin{abstract}
Lower hybrid (LH) drive involves the resonant acceleration of electrons parallel to the magnetic field by lower hybrid waves, often driven by ions with ring or ring-beam distributions. Charge-exchange between hydrogen atoms and protons with relative motions perpendicular to the magnetic field leads to ring distributions of pickup ions, with concomitant perpedicular ion 'heating'. This paper considers the combination of LH drive and charge-exchange in the outflow regions of magnetic reconnection sites in the solar chromosphere and lower corona, showing that the combined mechanism naturally predicts major perpendicular ion heating and parallel electron acceleration, and exploring the mechanism's relevance to specific solar reconnection phenomena, heating of the solar atmosphere, and production of energetic electrons that generate solar radio emission. Although primarily qualitative, analysis shows that the mechanism has numerous attractive aspects, including perpendicular ion heating that increases linearly with ion mass, parallel electron acceleration, predicted ion and electron temperatures that span those of the chromosphere and lower corona, and parallel electron speeds spanning those for type III bursts. Applications to chromospheric explosive events and low-lying active regions, and to heating the chromosphere, appear particularly suitable. Sweeping of plasma frozen-in to chromospheric and coronal magnetic field lines across the neutral atmosphere due to motions of sub-photospheric fields represents an obvious and important generalisation of the mechanism away from reconnection sites. The requirements that the neutrals not be strongly collisionally coupled to the plasma and that sufficient neutrals are available for charge-exchange restricts the LH drive mechanism to above the photosphere but below where the corona is essentially fully ionised. LH drive may thus be important in heating the chromosphere and low corona while other heating mechanisms dominate at higher altitudes. Although attractive thus far, quantitative analyses of LH drive in these contexts are necessary before definitive conclusions are reached.
\end{abstract}

Keywords: Sun: magnetic fields — Sun: activity — Sun: chromosphere — waves

\section{Introduction}

Magnetic reconnection in solar flares, magnetic loops, and helmet streamers has long been proposed (Parker 1963) as the 'first-phase' acceleration process that produces the semi-relativistic electrons responsible for type III solar radio bursts (Wild 1950; Melrose 1980; McLean \& Labrum 1985) and impulsive hard X-ray bursts (Dennis 1985; Tandberg-Hanssen \& Emslie 1988). Magnetic reconnection involves regions with antiparallel magnetic fields $\mathbf{B}$ being brought together at subAlfvénic speeds, with the reconnected magnetic flux and plasma being jetted outwards at the ends of the structure (Figure 1) at the Alfvén speed $V_{A}$. Theories for coronal heating also appeal to magnetic reconnection, for example in nanoflares (Parker 1990). But exactly how reconnection might produce these semi-relativistic electrons (speeds $\gtrsim 0.1 c$ ) and heated plasma is not clear. Specific problems include: (1) Reconnection theory constrains the exiting bulk speed of both electrons and ions to be $\lesssim V_{A}$, which is widely quoted as $\lesssim 3000 \mathrm{~km} \mathrm{~s}^{-1}\left(10^{-2} c\right)$ for the solar corona and lower regions. While localised regions with $v_{A} \gtrsim 0.1 c$ doubtless exist, they are not believed to be representative of reconnection regions. (2) Theoretically, reconnection primarily involves conversion of magnetic energy into bulk flow energy, not bulk heating. (3) Semi-relativistic electrons and significant plasma heating are not observed in or near magnetic reconnection sites at Earth's magnetopause (Phan et al. 2000). Accordingly, additional mechanisms must be active in reconnection sites to explain first-phase electron acceleration and plasma heating. The standard ones are (1) acceleration by parallel electric fields (Holman 1985; Benz 1987) and (2) stochastic acceleration via wave-particle interactions, which are reviewed in detail elsewhere (Miller et al. 1997).

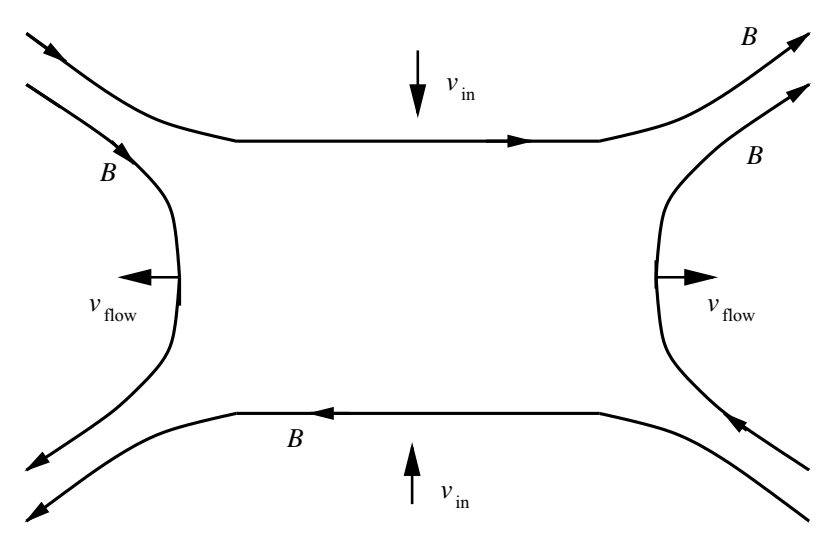

Figure 1 Schematic of an X-type reconnection region. 
Lower hybrid (LH) drive (McBride et al. 1972) involves lower hybrid waves resonantly accelerating electrons parallel to $\mathbf{B}$, as well as heating ions perpendicular to $\mathbf{B}$. The mechanism has been developed extensively for multiple laboratory and space contexts, with several sources for the lower hybrid waves, including cross-field currents (Spicer, Benz, \& Huba 1981; Benz \& Smith 1987) and modified two-stream instabilities involving perpendicular ion beams or rings (McBride et al. 1972; Papadopoulos 1984; Omelchenko et al. 1989; Cairns \& Gurnett 1991a; McClements et al. 1993; Shapiro et al. 1998; Cairns \& Zank 2001). McClements et al. (1993) proposed that LH drive might be relevant to first-phase acceleration of electrons and ions in solar hard $\mathrm{X}$ ray and gamma ray observations, with the ion ring distributions driving the lower hybrid waves. Their calculations suggest that the mechanism is viable in the corona. However, they did not rigorously identify the source of the required ion ring distributions or apply the mechanism to type III bursts or solar heating.

Recently, however, Wu (1996), Wu et al. (1998), and Wang et al. (2001) considered collisional charge-exchange of neutral hydrogen with protons in the outflow regions of solar magnetic reconnection sites. They showed that this 'pickup' process naturally forms ion ring distributions, as described in the next section. Wang et al. (2001) assumed low plasma $\beta$ (equal to the ratio of thermal to magnetic pressures) $\lesssim 10^{-2}$ and discussed the growth of MHD waves and associated scattering of the ring ions onto a shell in velocity space. They claimed reasonable agreement with the acceleration timescales and ion energies inferred from observations.

The crucial point recognised and elaborated upon here is that charge-exchange in low $\beta$ plasmas predominantly leads to the ion ring driving lower hybrid waves, which can then yield parallel electron acceleration through LH drive in addition to perpendicular ion heating. This has been observed near the space shuttle in Earth's ionosphere (Papadopoulos 1984; Cairns \& Gurnett 1991a), is well known theoretically (McBride et al. 1972; Omelchenko et al. 1989; Shapiro et al. 1998), and has been proposed for the $2-3 \mathrm{kHz}$ radio emissions generated beyond the heliospheric termination shock (Cairns \& Zank 2001). Accordingly, in Section 2 we generalise $\mathrm{Wu}$ and collaborators' scenario of charge-exchange in magnetic reconnection regions to include growth of lower hybrid waves and subsequent electron acceleration by LH drive, thereby placing McClements et al.'s (1993) work in a natural context. We demonstrate that this scenario naturally yields significant parallel electron acceleration and perpendicular ion heating. In Section 3 we apply this scenario to electron acceleration in the solar chromosphere and corona, including type III bursts, showing its attractive features but emphasising potential difficulties - the principal one being the requirement that a significant number of neutrals be present in the reconnection region. The heating and energy budgets of the chromosphere and corona still require detailed explanation. Accordingly in Section 4 we discuss LH drive in connection with solar heating, showing that $\mathrm{LH}$ drive can provide an alternative to, or a first phase for, recent wave heating models which involve ion cyclotron waves (McKenzie, Banaszkiewicz, \& Axford 1995; Cranmer 2000). In Section 5 it is pointed out that LH drive may also be important in solar contexts other than reconnection regions, in particular where subphotospheric motions drag plasma at chromospheric and higher altitudes across the neutral atmosphere, since the major requirement of $\mathrm{LH}$ drive is only that a perpendicular drift exist between plasma ions and neutrals. This section also contains a discussion of the paper's results. The paper's conclusions are in Section 6.

Before proceeding it is stated that the emphasis in this paper, despite applying LH drive in some detail to electron acceleration and electron/ion heating in the chromosphere and corona, is on presenting the ideas in a primarily qualitative fashion that will motivate further future development and discussion. No claim is made to a definitive analysis. Instead, while the paper's analyses show that LH drive appears attractive for a variety of phenomena, detailed work will be required before definitive conclusions can be reached.

\section{LH Drive in Magnetic Reconnection Regions}

Figure 1 is a schematic of an X-type reconnection region. Plasma carrying frozen-in magnetic field lines convects towards the neutral line, with antiparallel field directions on either side. Convection is at the $\mathbf{E} \times \mathbf{B}$ drift velocity associated with a convection electric field directed into the page. Within a few ion Larmor radii of the neutral sheet's centre, in the so-called diffusion region, the frozen-in assumption breaks down, the magnetic field lines partially annihilate but also reconnect, and the plasma is accelerated outward along the neutral sheet axis by magnetic forces. Standard energy and mass balance arguments imply that the outflow has a speed of order $V_{A}$. Since the outflow velocity $\mathbf{v}_{\text {flow }}$ must be perpendicular to $\mathbf{B}$ on the axis, by symmetry, the outflow must also be an $\mathbf{E} \times \mathbf{B}$ drift. Off-axis, $\mathbf{v}_{\text {flow }}$ may have a parallel component too.

Collisional charge-exchange of neutral hydrogen atoms with the outflowing protons means that neutrals instantaneously become protons with the same initial velocity $\mathbf{v}_{0}$ while the outflowing protons become neutrals with velocity $\mathbf{v}_{\text {flow }}$, assumed perpendicular to $\mathbf{B}$ for now. This situation leads naturally to ion ring distributions ( $\mathrm{Wu}$ 1996; Wu et al. 1998; Wang et al. 2001): in the rest frame of the outflowing protons there is no convection electric field but a newborn proton starts off at $\mathbf{v}=\mathbf{v}_{\perp}=-\mathbf{v}_{\text {flow }}+\mathbf{v}_{0}$ and so gyrates with gyrospeed $v_{g}=v_{\perp}=\left|-\mathbf{v}_{\text {flow }}+\mathbf{v}_{0}\right|$, thereby being found on a ring in perpendicular (to $\mathbf{B}$ ) velocity space with ring speed

$$
v_{r}=v_{\text {flow }} .
$$

Viewed instead in the rest frame of the neutral sheet, the newborn proton starts off with perpendicular velocity $\mathbf{v}_{0}$ but experiences both the convection electric field and magnetic forces, developing an $\mathbf{E} \times \mathbf{B}$ drift velocity equal 

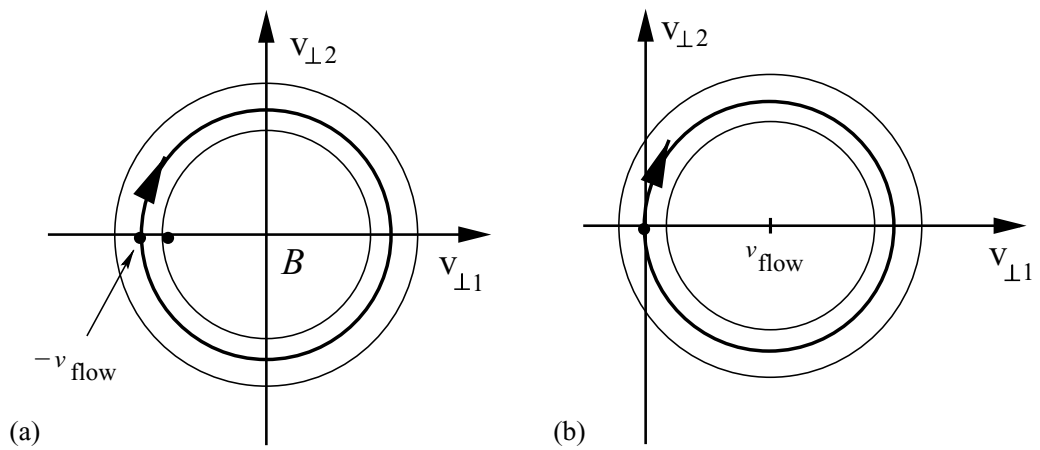

Figure 2 Perpendicular phase space plots of pickup ion distributions for $\mathbf{v}_{\text {flow }}$ perpendicular to $\mathbf{B}$. The heavy circle shows the gyroorbit and pickup ring for a pickup ion with $\mathbf{b}_{0}=0$, the inner and outer circles are gyroorbits for different $\mathbf{v}_{0}$, and the arrows on the pickup ring show the sense of gyration for $\mathbf{B}$ directed out of the page. (a) In the outflow frame, the ring is centred at zero velocity. (b) In the rest frame of the neutrals, the ring is centred at $\mathbf{v}_{\text {flow }}$. See the text for more details.

to $\mathbf{v}_{\text {flow }}$ and a gyrospeed $v_{g}$. Accordingly, the newborn ions are convected with the ambient plasma flow at $\mathbf{v}_{\text {flow }}$ (thus the name 'pickup ions'), have a large gyrospeed $\approx v_{\text {flow }}$ in the case $v_{\text {flow }} \gg v_{0}$ (e.g. a small thermal speed compared with $v_{\text {flow }}$ ) that mimics perpendicular heating, and are found on a ring in perpendicular velocity space (Figure 2). Provided then that the charge-exchange frequency is much smaller than the gyrofrequency, the newborn ions have a ring distribution (with equal phase space densities around the ring). In the opposite limit the ions have a beam distribution centred near $\mathbf{v}_{0}$ in the neutral sheet frame, with beam-arc distributions for intermediate cases (Cairns 1990). Allowing $\mathbf{v}_{\text {flow }}$ to have a component parallel to $\mathbf{B}$ introduces a relative neutral-plasma drift along $\mathbf{B}\left(=\mathbf{v}_{\text {flow }} \cdot \mathbf{B} \mathbf{B} / B^{2}\right)$, giving the pickup ions ringbeam distributions rather than pure ring distributions, and the ring speed becomes the magnitude of the perpendicular component of $\mathbf{v}_{\text {flow. }}$.

The development of these ring distributions of pickup ions therefore involves only simple kinematics and chargeexchange. However, the plasma must supply the kinetic energy of the pickup ions despite losing the kinetic energy of the new neutral particles; this energy comes from the convection electric field and the plasma outflow, thereby having its origin in the overall reconnection process. Note that the apparent perpendicular heating of pickup ions is to the very considerable temperature

$$
k_{B} T_{\perp i} \approx m_{i} v_{r}^{2} \approx m_{i} V_{A}^{2},
$$

where $k_{B}$ is Boltzmann's constant and $m_{i}$ is the ion mass. Figure 3 shows the substantial perpendicular heating predicted by (2) as a function of $v_{r}$ and/or $V_{A}$. This prediction likely breaks down as $v_{r}$ approaches $c$ (indicated by the dashed line) due to neglect of relativistic effects. However, as $v_{A}$ increases from 10 to $2000 \mathrm{~km} \mathrm{~s}^{-1}$ (plausible numbers for the chromosphere and corona), (2) predicts that $T_{\perp i}$ increases from $10^{4}$ to $5 \times 10^{8} \mathrm{~K}$, thereby covering the range of ion temperatures observed in the chromosphere and corona (Kohl et al. 1997). Equation (2) predicts, moreover, that $T_{\perp i}$ is proportional to the ion mass

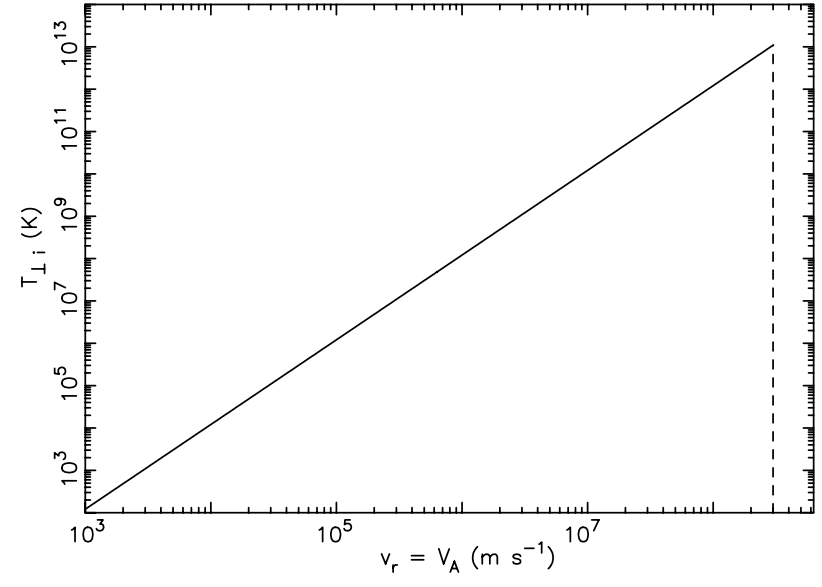

Figure 3 The prediction (2) for $T_{\perp i}$ as a function of $v_{\text {flow }}=V_{A}$ for the range of $V_{A}$ encountered in the Sun's atmosphere. Dashed lines at $v_{\text {flow }}=c$ show where the prediction breaks down.

$m_{i}$ for LH drive, but not to the ion charge. This prediction is directly relevant to observations (Kohl et al. 1997) of much greater oxygen ion heating than proton heating, since from (2), $T_{\perp O} \approx 16 T_{\perp H}$.

Perpendicular ring, ring-beam, and beam distributions of ions are often unstable to the growth of lower hybrid waves (McBride et al. 1972; Papadopoulos 1984; Omelchenko et al. 1989; Cairns \& Gurnett 1991a; McClements et al. 1993; Shapiro et al. 1998). These waves are primarily electrostatic and propagate primarily perpendicular to $\mathbf{B}$. Their wavenumbers perpendicular $\left(k_{\perp}\right)$ and parallel $\left(k_{\|}\right)$to $\mathbf{B}$ are related by

$$
k_{\|} / k_{\perp} \approx(0.5-1) \sqrt{\frac{m_{e}}{m_{i}}},
$$

where $m_{e}$ is the electron mass. The waves have frequencies close to the lower hybrid frequency $\omega_{L H} \approx \Omega_{i} \sqrt{m_{i} / m_{e}}$, where $\Omega_{i}$ is the proton gyrofrequency. For a ring instability the resonance condition for lower hybrid waves then implies

$$
\omega=k_{\perp} v_{r} \approx \omega_{L H}
$$


(An almost identical condition occurs when a perpendicular beam drives lower hybrid waves via the so-called modified two-stream instability (McBride et al. 1972; Papadopoulos 1984; Cairns \& Gurnett 1991b), with replacement of $v_{r}$ by the relative perpendicular beam speed.) Growth of lower hybrid waves takes energy out of the ring, broadening and moving it to lower speeds by quasilinear relaxation (McBride et al. 1972; Omelchenko et al. 1989). However, randomness in the evolving wave spectrum also allows resonant acceleration of some ions into a tail that extends to higher energies (McBride et al. 1972; Omelchenko et al. 1989; McClements et al. 1993).

$\mathrm{LH}$ drive involves resonant electron acceleration to large parallel speeds $v_{\| e}$ due to the rapid movement of the wave phase fronts along $\mathbf{B}$. The Cherenkov resonance condition then yields

$$
\omega=k_{\|} v_{\| e} .
$$

Then, combining (1) with (4) with $v_{\text {flow }}=v_{A}$ implies that LH drive in reconnection regions can produce electrons with

$$
\begin{aligned}
v_{\| e} & \approx(1-2) \sqrt{\frac{m_{i}}{m_{e}}} V_{A}, \\
& \approx(40-90) V_{A}
\end{aligned}
$$

for neutral hydrogen. More complete analyses (McBride et al. 1972; Omelchenko et al. 1989; Shapiro et al. 1998) show that LH drive pulls a superthermal tail out of the ambient electron tail, typically starting from a few thermal speeds $V_{e}$ and extending to $\approx v_{\| e}$. Approximating the tail as being flat $\left(\partial f / \partial v_{\|}=0\right)$, it is easy to show from (6) that the effective parallel temperature of the accelerated electrons is

$$
k_{B} T_{\|, e}=m_{e}\left\langle v_{\|}^{2}\right\rangle \approx(1-4) \frac{m_{i} V_{A}^{2}}{3} .
$$

Comparing (2) and (8), this mechanism yields approximately equal perpendicular ion heating and parallel electron heating.

Figure 4 shows the significant parallel electron acceleration predicted by (6) as a function of $v_{r}=V_{A}$. Note that the prediction (6) should break down when either $v_{r}$ or $v_{\| e}$ approaches $c$, due to neglect of relativistic effects. Nevertheless, it is clear that for plausible values of $v_{A}$ in the chromosphere and corona $\left(\approx 10-2000 \mathrm{~km} \mathrm{~s}^{-1}\right),(6)$ predicts that $v_{\| e}$ spans the range $4 \times 10^{5}-1.8 \times 10^{8} \mathrm{~m} \mathrm{~s}^{-1}$, while (8) predicts $T_{\| e} \approx 10^{4}-10^{8} \mathrm{~K}$. These numbers are attractive in several solar contexts, as explained in more detail in Sections 3 and 4.

\section{Application to Solar Electron Acceleration and Type III Bursts}

This section applies the foregoing theory for chargeexchange and LH drive in reconnection outflow regions to the parallel acceleration of electrons and perpendicular energisation of ions in the solar atmosphere. Attention

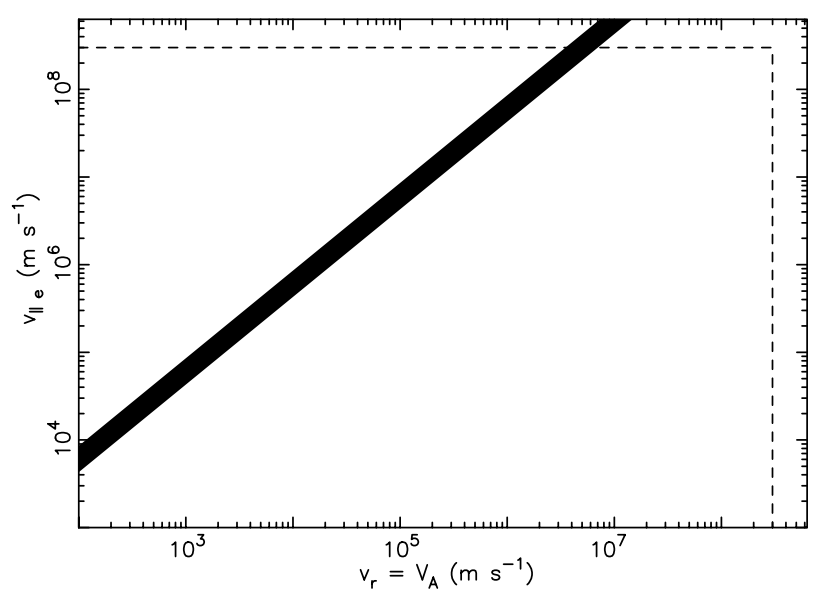

Figure 4 The prediction (6) for $v_{\| e}$ as a function of $v_{\text {flow }}=V_{A}$ for the range of $V_{A}$ encountered in the Sun's atmosphere. Dashed lines at $v_{\text {flow }}=c$ and $v_{\| e}=c$ show where the prediction breaks down.

is focused first on electron acceleration in chromospheric and transition region phenomena which show evidence for reconnection flows, then on low-lying solar active regions, and then on higher coronal events at heights of order 0.1 solar radii that may be relevant to type III bursts. The ion energisation produced in LH drive is compared with the scenario of Wu and colleagues (Wu 1996; Wu et al. 1998; Wang et al. 2001) in Section 3.4.

\subsection{Chromospheric and Transition Region Events}

So-called 'explosive events' are small-scale explosions or microflares that occur near boundaries of the quietSun chromospheric network (Brueckner \& Bartoe 1983; Dere, Bartoe, \& Brueckner 1989; Dere 1994; Innes et al. 1997a,b; Innes \& Toth 1999). They are frequent $(\approx 3000$ occurring over the Sun's surface at one time), shortlived (durations $\approx 60 \mathrm{~s}$ ), and have small spatial scales $(\approx 2000 \mathrm{~km})$. Their most important characteristics here, however, are (1) their large blue and/or red Doppler shifts in emission lines consistent with chromospheric and transition region temperatures $\left(\approx 2 \times 10^{4}-5 \times 10^{5} \mathrm{~K}\right)$ and inferred speeds $\sim 100 \mathrm{~km} \mathrm{~s}^{-1}$, and (2) their spatial structure being consistent with bidirectional flows (Innes et al. 1997a,b). Explosive events are therefore directly interpreted in terms of magnetic reconnection events at network boundaries, not inconsistent with the enhanced magnetic activity observed there.

Supposing only that the relative number density of neutral hydrogen atoms $\left(n_{H}\right)$ to electrons $\left(n_{e}\right)$ is significant in these regions, then Section 2's model appears qualitatively suited for modelling explosive events. This assumption appears very plausible in view of ionisation balance calculations and associated observational comparisons for the solar chromosphere and transition region (Gabriel 1971; Fontenla, Avrett, \& Loeser $1990,1991,1993)$, which imply that the ratio $n_{H} / n_{e}$ is $\gtrsim 1$ for $T_{e}=T_{i} \lesssim 8000 \mathrm{~K}, \approx 10^{-2}$ for $5 \times 10^{4} \mathrm{~K}$, and $\approx 2 \times 10^{-5}$ for $10^{5} \mathrm{~K}$. Similar calculations and observations for the corona imply that $n_{H} / n_{e} \approx(1-10) \times 10^{-7}$ 
for $T_{e}=(1-2) \times 10^{6} \mathrm{~K}$ (Shklovskii 1965; Gabriel 1971; Fontenla et al. 1990, 1991, 1993; Cranmer 2000). Moreover, since the collision frequency decreases rapidly above the photosphere, so that kinetic effects should be included rather than assuming fluid theory and very small collisional mean free paths relative to plasma scale lengths, and since the chromosphere is likely not in equilibrium due to the dynamic effects of spicules, explosive events, EUV blinkers (Harrison 1997; Harrison et al. 1999), and magnetic activity, even higher levels of neutrals can be expected, at least sometimes, in these regions.

Inserting the flow speeds $\sim 100 \mathrm{~km} \mathrm{~s}^{-1}$ of explosive events into (6) immediately implies (Figure 4) that $\mathrm{LH}$ drive should produce electrons with $v_{\| e} \approx 6 \times 10^{6} \mathrm{~m} \mathrm{~s}^{-1}$, corresponding to about 10 electron thermal speeds $V_{e}$ for an assumed chromospheric electron temperature of $2 \times 10^{4} \mathrm{~K}$. Theoretically, these superthermal electrons may couple effectively to the background plasmas if they develop beam distributions (parallel to $\mathbf{B}$ ) and drive Langmuir waves, whose energy is then converted either into radio emissions or increased thermal plasma energy by damping. These beam speeds, $\lesssim 10^{7} \mathrm{~m} \mathrm{~s}^{-1}$, are much less than those inferred from the drift rates of solar decimetric type III bursts (Aschwanden, Benz, \& Schwartz 1993; Benz, Csillaghy, \& Aschwanden 1996). However, based on both remote and in situ wave observations the average exciter speed of interplanetary type III bursts is $\approx 0.14 c \approx 4.5 \times 10^{7} \mathrm{~m} \mathrm{~s}^{-1}$ (Dulk et al. 1987), with minimum and maximum values of $\approx 10^{7} \mathrm{~m} \mathrm{~s}^{-1}$ and $\approx 10^{8} \mathrm{~m} \mathrm{~s}^{-1}$ (Dulk et al. 1987; Hoang, Dulk, \& Leblanc 1994), respectively. Accordingly, LH drive in explosive events can produce electrons with parallel speeds approaching those required for at least some interplanetary type III bursts.

Significant acceleration is not expected from all chromospheric and transition region events. Consider, for instance, spicules in the chromosphere and EUV blinkers observed in transition region lines (Harrison 1997; Harrison et al. 1999). The former involve upward but probably field-aligned flows at $\approx 20-30 \mathrm{~km} \mathrm{~s}^{-1}$ with unknown origin, while the latter are believed to involve reconnection near boundaries of the chromospheric network but show no evidence for significant flows above the instrument resolution of $\lesssim 25 \mathrm{~km} \mathrm{~s}^{-1}$. If spicule flows are parallel to B then charge-exchange will give rise to parallel beams, not ion rings, so that $\mathrm{LH}$ drive will not apply. This context is discussed more in Section 5. For EUV blinkers, however, (6) implies $v_{\| e} \lesssim 10^{6} \mathrm{~m} \mathrm{~s}^{-1}$. These events are thus expected to be less important than explosive events.

\subsection{Chromosphere and Low Corona: Low-lying Active Regions}

Consider next reconnection at the footpoints of magnetic loops, as often inferred from $\mathrm{H} \alpha$ and $\mathrm{X}$ ray data. In these regions $V_{A}$ plausibly reaches $\approx 1000-2000 \mathrm{~km} \mathrm{~s}^{-1}$, whence (6) and Figure 4 predict $v_{\| e}$ in the range $\approx(0.4-1.8) \times 10^{8} \mathrm{~m} \mathrm{~s}^{-1}$ or $\approx(0.1-0.6) c$. These electrons can then develop beam distributions by time-of-flight effects with speeds in the range $\approx(0.1-0.6) c$ typically observed for coronal and interplanetary type III bursts (Wild 1950; Melrose 1980; McLean \& Labrum 1985; Dulk et al. 1987; Hoang et al. 1994). Stochastic or parallel electric field acceleration could further increase these speeds by another factor of 2 or more, in order to explain some type III bursts with beam speeds very close to $c$ (Poquerusse 1994). In principle, then, LH drive appears an attractive mechanism for producing electron beams from low-lying reconnection regions that generate type III bursts. One proviso is, however, that sufficient numbers of neutral hydrogen atoms are available. As argued above in Section 3.1, the neutral hydrogen fraction should be in excess of $10^{-6}$ for chromospheric and photospheric flares, only arguably being smaller than this in the corona proper.

\subsection{High Coronal Acceleration Events Involving Reconnection}

Some flares and associated sites of reconnection and electron beam formation appear to be at altitudes $\approx 0.1 R_{S}$ or $\approx 70000 \mathrm{~km}$, corresponding to the corona itself rather than the chromosphere or transition region. In these regions typical estimates for $V_{A}$ are $\approx 400-2000 \mathrm{~km} \mathrm{~s}^{-1}$, whence (6) and Figure 4 predict production of electrons with $v_{\| e} \approx(0.05-0.5) c$ by LH drive. These appear attractive for type III bursts, especially if stochastic acceleration or parallel electric fields in the reconnection region further accelerate the electrons, provided the following basic question can be answered affirmatively: Do large enough concentrations of neutrals exist in these regions for the pickup ion/LH drive process to proceed effectively? If they do (say, $n_{n} / n_{e} \gtrsim 10^{-6}$ ), then LH drive appears viable. If not, then alternative mechanisms must apply.

It appears that this question cannot be answered definitively at present. Reasons for this are: (1) Ionisation calculations for the corona (Shklovskii 1965; Gabriel 1971; Fontenla et al. 1990, 1991, 1993; Cranmer 2000), balancing collisional ionisation with radiative recombination, yield ratios $n_{n} / n_{e} \approx 2 \times 10^{-5}$ for $T_{e}=10^{5} \mathrm{~K}$ and $\approx(0.1-1) \times 10^{-6}$ for $T_{e} \gtrsim 10^{6} \mathrm{~K}$. (2) The observed properties of prominences imply that significant amounts of neutral gas with $T \lesssim 8000 \mathrm{~K}$ can persist stably to such altitudes, in which case the ionisation balance calculations yield $n_{w} / n_{e} \approx 1$ (Fontenla et al. 1990, 1991, 1993), albeit without satisfactory theoretical explanation. (3) To my knowledge, kinetic calculations of ion, electron, and neutral distribution functions coupled by charge-exchange, Coulomb, and ordinary collisions, radiative ionisation and recombination do not yet exist for the transition from the collisional photosphere to the collisionless corona even in the steady state limit, let alone the nonequilibrium limit that includes the effects of spicules, magnetic activity, network evolution, multiple generations of charge-exchange, etc. Thus, even though these altitudes correspond to about 100 hydrostatic gravitational scale heights for the chromosphere $\left(\approx 10^{-3}\right.$ solar radii $)$, it appears that LH drive cannot 
be ruled out for these high reconnection sites. Nevertheless, while further analysis of the neutral density is required, at present the combination of $\mathrm{LH}$ drive with pickup ions appears relatively unlikely to explain electron energisation in most coronal type III bursts.

\subsection{Ion Acceleration in These Regions}

The pickup process primarily increases the gyrospeed $v_{\perp}$ of the pickup ions to $v_{\text {flow }}$ but preserves the original parallel speed of the neutral atom, while also leading to $\mathbf{E} \times \mathbf{B}$ convection with the rest of the plasma. The LH drive process primarily involves the pickup ring being broadened, mostly to small $v_{\perp}$ although with a small tail to larger $v_{\perp}$ due to stochastic acceleration (McBride et al. 1972; Omelchenko et al. 1989; McClements et al. 1993; Shapiro et al. 1998), but does not affect $v_{\|}$. This is a significant difference from the situation studied by $\mathrm{Wu}$ and collaborators (Wu 1996; Wu et al. 1998; Wang et al. 2001), in which growth of Alfvénic waves by pickup ions leads to scattering onto a shell in velocity space and so to significant increases in $v_{\| i}$. Instead, LH drive with pickup ions leads to perpendicular ion acceleration only to about 4 times the flow energy, to a perpendicular energy $\approx 2 m_{i} v_{\text {flow }}^{2} \approx 2 m_{i} V_{A}^{2}$. However, this process corresponds to very effective perpendicular ion heating (Section 4).

The negligible amount of parallel ion acceleration produced by LH drive is important in connection with firstphase acceleration, which is preferentially observed to produce energetic electrons in the solar wind (Miller et al. 1997). Relevant points are that the gravitational escape speed is $v_{\mathrm{esc}} \approx 6 \times 10^{5} \mathrm{~m} \mathrm{~s}^{-1}$ and that only particles with parallel speeds in excess of $v_{\text {esc }}$ can reach the solar wind. Accordingly, LH drive is expected to produce negligible ion acceleration in the solar wind, since the acceleration is primarily perpendicular to $\mathbf{B}$ and the initial gas speeds (and so $v_{\|}$) are small compared with $v_{\text {esc }}$, but significant parallel electron heating. It thus appears that LH drive is a viable mechanism for the first-phase acceleration process.

\section{Application to Heating in Solar Reconnection Regions}

Consider now the ion and electron 'heating' predicted for LH drive by (2) and (8), respectively, in the contexts discussed in Section 3 for particle acceleration.

\subsection{Chromospheric and Transition Region Events}

For chromospheric explosive events (Innes et al. 1997a,b), inserting the inferred speeds of $\approx 100 \mathrm{~km} \mathrm{~s}^{-1}$ into (2) and (8) implies that $T_{\perp i} \approx 1.2 \times 10^{6} \mathrm{~K}$ and $T_{\| e} \approx(0.4-1.6) \times$ $10^{6} \mathrm{~K}$ (Figure 3 ). The inferred heating is therefore very large, being well in excess of the chromospheric temperatures $\approx(2-5) \times 10^{4} \mathrm{~K}$. However, the ion plasma temperature observed will be weighted by the number density of each ion population in the plasma, that is,

$$
\left\langle T_{\mathrm{obs}}\right\rangle \approx \Sigma n_{\alpha} T_{\alpha} / \Sigma n_{\alpha}
$$

where the sum is over all ion populations $\alpha$ in the plasma. A similar equation follows for the observed electron temperature. Comparing the predictions of (2) to the temperatures observed for explosive events, $\approx(0.2-5) \times 10^{5} \mathrm{~K}$ (Dere 1994), consistency is obtained if pickup ions typically represent only a fraction $\approx 10^{-2}-0.4$ of the total plasma density. This seems reasonable based on equilibrium ionisation balance calculations for the chromosphere (Gabriel 1971; Fontenla et al. 1990, 1991, 1993), which yield $n_{w} / n_{e} \approx 10^{-2}-1$ (Section 3.1).

For EUV blinkers (Harrison 1997; Harrison et al. 1999), with $v_{\text {flow }} \lesssim 25 \mathrm{~km} \mathrm{~s}^{-1}$, the predictions (2) and (8) yield $T_{\perp i} \approx 8 \times 10^{4} \mathrm{~K}$ and $T_{\| e} \approx(3-10) \times 10^{4} \mathrm{~K}$. These temperatures are very characteristic of the chromosphere and transition region, although as for explosive events, reweighting via (9) will reduce the predicted heating. Inserting the flow speeds for spicules into the above formulae (despite spicule flows likely being parallel to B) yields very similar results to EUV blinkers.

Noting that current models encounter theoretical difficulties in explaining the chromosphere's relatively large temperature compared with the photosphere, it appears that $\mathrm{LH}$ drive in reconnection regions can produce ion (and electron) temperatures and associated apparent heating with large enough magnitudes to be a major contributor to the chromosphere's temperature and energy budget. LH drive may, of course, coexist with other mechanisms. Generalisation of this mechanism to heating outside reconnection sites is discussed in Section 5.

\subsection{Low-lying Active Regions: Chromosphere and Low Corona}

The large Alfvén speeds characteristic of reconnection sites at the chromospheric or low-coronal footpoints of active-region loops should lead to very large heating if LH drive is active: inserting $V_{A}=400$ and $2000 \mathrm{~km} \mathrm{~s}^{-1}$ into (2) and (8) leads to (Figure 3) $T_{\perp i}=2 \times 10^{7} \mathrm{~K}$ and $5 \times 10^{8} \mathrm{~K}$, and $T_{\| e}=(0.7-3) \times 10^{7} \mathrm{~K}$ and $(2-6) \times 10^{8} \mathrm{~K}$, respectively. Thus, $\mathrm{LH}$ drive predicts that regions with larger $V_{A}$ should have larger temperatures, qualitatively consistent with observations that regions with larger magnetic fields and magnetic activity have larger temperatures. In addition, $\mathrm{LH}$ drive predicts that the ion temperature should be primarily perpendicular, rather than isotropic or primarily parallel, as observed recently (Kohl et al. 1997). The numerical values for $T_{\perp i}$ and the prediction (2) that $O^{5+}$ ions should have much higher $T_{\perp}$ than protons (by a factor of the mass ratio) are also consistent with the data (Kohl et al. 1997).

It is relevant, however, that averaging over plasma components is appropriate, cf. (9), which will lower $\left\langle T_{\mathrm{obs}}\right\rangle$ compared with the prediction (2). Since Section 3.1's discussion of ionisation balance calculations and observations (Gabriel 1971; Fontenla et al. 1990, 1991, 1993) implies that $n_{w} / n_{e}$ has a very wide range $\left(\approx 2 \times 10^{-5}-1\right)$ for chromospheric and transition region temperatures, detailed data for specific events are required to test the theory in any detail. At present, however, LH drive appears 
an attractive theory that is qualitatively consistent with available data.

\subsection{High Coronal Reconnection Sites}

Coronal reconnection sites at heights $\lesssim 0.1 R_{S}$ are expected to have $V_{A}$ in the same range (400-2000 $\mathrm{km} \mathrm{s}^{-1}$ ) considered in Section 4.2 for low-lying active regions. Accordingly, Section 4.2's estimates for $T_{\perp i}$ and $T_{\| e}$ and their associations with magnetic activity and ion mass follow here too.

The ratio $n_{w} / n_{e}$ of neutral hydrogen strongly determines whether these temperature increases would be observable: if $n_{w} / n_{e} \lesssim 10^{-6}$, as predicted by the static, fluid ionisation balance calculations (Shklovskii 1965; Gabriel 1971; Fontenla et al. 1990, 1991, 1993; Cranmer 2000) discussed in Sections 3.1 and 3.3, then (9) predicts that LH drive will not produce observable temperature enhancements at large coronal heights. Indeed, only if $n_{w} / n_{e} \gtrsim 10^{-2}$ and $v_{A} \gtrsim 1000 \mathrm{~km} \mathrm{~s}^{-1}$, would $\left\langle T_{\mathrm{obs}}\right\rangle$ increase significantly above the local temperature $\approx 10^{6} \mathrm{~K}$. Limitations to our current knowledge of $n_{w} / n_{e}$ are listed in Section 3.3. Nevertheless, while definite conclusions on the relevance of $\mathrm{LH}$ drive for plasma heating in the corona cannot be reached currently, the requirement $n_{w} / n_{e} \gtrsim 10^{-2}$ renders the mechanism unlikely.

\section{Generalisations and Discussion}

The calculations in Sections 2-4 suggest that LH drive due to collisional charge-exchange of neutral hydrogen in the outflow regions of solar reconnection regions is attractive for producing significant perpendicular ion heating and parallel electron acceleration in explosive events and low-lying reconnection events in the chromosphere and (less plausibly) lower corona. In particular, the mechanism can plausibly produce ions and electrons with the range of perpendicular and parallel, respectively, temperatures observed in the chromosphere, transition region, and low-lying active regions, as well as electrons with parallel speeds and effective parallel temperatures close to those inferred for type III bursts. The LH drive mechanism also predicts that higher mass ions should have perpendicular temperatures greater by the mass ratio from protons. However, the mechanism is most likely not applicable in high coronal reconnection sites, due to requirements on $n_{w} / n_{e}$, although it is not definitely ruled out. Importantly, the mechanism is not inconsistent with the small plasma heating and electron acceleration observed near Earth's magnetopause, where magnetic reconnection is definitely observed (Phan et al. 2000), since the number density of neutrals is minimal there. The calculations and discussion above are relatively qualitative and should not be considered definitive. However, the theory appears attractive and should be developed further. Significantly, though, the LH drive/pickup ion mechanism potentially has significantly wider applicability, as discussed next.

LH drive can apply in other contexts where a plasma moves relative to neutrals with a velocity component perpendicular to $\mathbf{B}$, not only in reconnection regions. Since the solar atmosphere has very low plasma beta and the plasma is typically tied (or frozen) to magnetic field lines, movement of sub-photospheric fields will cause the field at higher altitudes to sweep the plasma back and forth across the neutral components of the atmosphere. Where the plasma and neutrals are tightly coupled, for instance where the collision frequency is large compared with the ion gyrofrequency, then charge-exchange collisions will primarily involve relabelling of particles (apart from a net 'pickup' current); charge-exchange will produce appreciably non-Maxwellian (ring) features in the plasma ion distributions only where the collision rate is relatively small, i.e. above the photosphere proper. That is, the ring-beam distributions required for LH drive to proceed, leading to particle acceleration and heating, can be produced only where the plasma and neutrals are starting to decouple collisionally, meaning in the chromosphere and above. This is qualitatively consistent with the solar atmosphere increasing in temperature above the photosphere.

Sweeping of magnetic field lines across the neutrals in the chromosphere and above is expected to be particularly relevant near network boundaries, active regions, and magnetic loops. Indeed with $V_{A}$ and the apparent sweeping speed typically increasing with altitude, the heating and particle acceleration produced by LH drive should initially increase substantially with altitude (Sections 3 and 4) before decreasing again as the ratio $n_{w} / n_{e}$ decreases for plasma temperatures characteristic of the transition region and above. This 'sweeping' mechanism for LH drive thus predicts that heating and particle acceleration should be particularly important in regions with significant magnetic activity, producing perpendicular ion and parallel electron temperatures quite characteristic of the chromosphere and transition region.

In addition, there are several 'heating-at-a-distance' effects that may be important for this sweeping mechanism. First, in the neutral rest frame, the neutral products of the charge-exchange have increased speed $\left(\approx v_{\text {flow }}\right)$ compared with the initial neutrals, thereby being able to reach greater heights. Second, the increase in $V_{A}$ and/or the bulk plasma (wind) speed with height means that picked-up protons will have larger ring speeds and so larger perpendicular heating and parallel electron acceleration with height. Third, multiple generations of charge-exchange are possible. These characteristics are attractive, suggesting that the combination of $\mathrm{LH}$ drive and the sweeping mechanism might be a significant heating/acceleration mechanism in at least the chromosphere and low corona, perhaps as the first stage of the overall mechanism heating the chromosphere and corona. These ideas should motivate further work.

Qualitatively, LH drive is attractive for heating the solar atmosphere since it naturally predicts perpendicular ion heating and parallel electron acceleration, as observed using spectroscopic measurements (Kohl et al. 1997) and as inferred from solar wind observations near $1 \mathrm{AU}$. Of course, more than one microphysical mechanism may 
contribute. For instance, the existing ion cyclotron heating model (McKenzie et al. 1995; Cranmer 2000) has similar predictions but does not consider the finite neutral hydrogen present or charge-exchange physics. The ion cyclotron mechanism is probably better suited to explaining heating higher in the transition region and corona than $\mathrm{LH}$ drive. However, the two may play complementary roles: LH drive may jumpstart the heating process at low heights while the ion cyclotron mechanism and others may work at larger heights.

It is worth emphasising that the enhanced parallel electron tail produced by LH drive is potentially relevant in other ways too. First, it resembles the superthermal electron tail required in the 'velocity filtration' explanation for the temperature profile of the corona and solar wind (Scudder 1992). Second, electrons streaming outward along open $\mathbf{B}$ field lines will develop an ambipolar electric field that drags the protons outward, potentially forming the solar wind. For LH drive, the bulk flow would thus develop at heights above the main heating/acceleration region in which LH drive occurs, not inconsistent with available scintillation and spectroscopic data. Since a superthermal ion tail develops during the wave growth phase (McBride et al. 1972; Omelchenko et al. 1989; McClements et al. 1993), the first point above also applies to the ions.

Before concluding, consider the effects of chargeexchange for spicules and other flows that are primarily parallel to $\mathbf{B}$. In this case, the charge-exchange leads to two ion beams separated by the relative parallel drift velocity between the neutrals and plasma. This situation is unstable to various streaming instabilities involving Alfvén and ion cyclotron waves, as reviewed elsewhere (Gary 1993). Wave-particle scattering and the back-reaction to the instability will thermalise, scatter and partially heat the ions, with the estimate (2) remaining appropriate after replacing $V_{A}$ with the drift speed. Second-order Fermi acceleration may also be relevant as these wave-particle interactions are well known to scatter the ions onto shells in velocity space. However, little electron heating or acceleration should result from these instabilities, due to only electrons with semi-relativistic speeds resonating with these waves (Melrose 1974), very different from the situation of LH drive with a perpendicular neutral-plasma drift.

\section{Conclusions}

The microphysical processes of charge-exchange, ion pickup, and LH drive lead naturally to perpendicular ion heating, parallel electron acceleration, and effective parallel electron heating. These processes require a source of new ions and a net drift perpendicular to the magnetic field between the plasma protons and the neutrals. In the solar contexts analysed in this paper the new ions are produced by collisional charge-exchange between hydrogen atoms and protons, while the perpendicular plasma-neutral drift is provided either by the outflowing plasma of magnetic reconnection jets or else by sub-photospheric motions causing the magnetic field at higher altitudes to sweep the frozen-in plasma across the neutrals.

LH drive's qualitative characteristics of perpendicular ion heating (with temperature proportional to the ion mass) and parallel electron acceleration (with parallel 'heating' manifested by an extended tail) are qualitatively consistent with the range of ion and electron temperatures observed in the solar atmosphere (Kohl et al. 1997; Miller et al. 1997), the dominance and mass dependence of perpendicular ion heating (Kohl et al. 1997), the temperature anisotropies of solar wind electrons and ions, and the predominance of electrons in first-phase accleration events like type III solar radio bursts (Miller et al. 1997). Moreover, the numerical analyses above suggest that the LH drive mechanism is likely relevant at least to explosive events in the chromosphere and to low-lying active regions in the chromosphere and (less plausibly) low corona where magnetic reconnection occurs; the sweeping mechanism may permit LH drive to be widely relevant in the chromosphere and perhaps the lower corona. The analyses suggest that LH drive with these two sources of perpendicular drifts may contribute significantly to the chromosphere's energy budget, heating the chromosphere and lower solar atmosphere, the production of electrons with similar parallel speeds to type III bursts, and generating significant nonthermal electron tails in the low solar atmosphere.

The requirements that significant neutrals be present and that the neutrals and ions not be strongly collisionally coupled restricts the regions where LH drive can proceed effectively to be above the photosphere but below altitudes where the medium is essentially fully ionised (say, $n_{H} / n_{e} \lesssim 10^{-6}$ ) like the nominal corona. This means that LH drive could be an important heating mechanism at low altitudes, such as the chromosphere and transition region, with ion cyclotron heating (McKenzie et al. 1995; Cranmer 2000) or other mechanisms dominating at higher altitudes. The analyses here are not definitive and require detailed evaluation, both in terms of comparing theoretical predictions with observations and in terms of determining the properties of neutrals thoughout the solar atmosphere, the latter specifically in flaring regions, the corona, and the acceleration sites for the electrons producing type III bursts. However, LH drive appears sufficiently attractive in a number of solar contexts that the mechanism should be evaluated in greater detail.

\section{Acknowledgments}

This paper was written for, and partially presented at, the Festschrift for Professor D. B. Melrose (DBM). The author strongly appreciates the guidance and help of DBM over the 19 years since starting his Honours thesis. Financial support from the Australian Research Council and NASA grant NAG5-7390 is gratefully acknowledged, as are useful discussions with S. Cranmer, P. Isenberg, and DBM, and comments on the manuscript by P. Robinson. 


\section{References}

Aschwanden, M. J., Benz, A. O., \& Schwartz, R. A. 1993, ApJ, 464, 985

Benz, A. O. 1987, Sol. Phys., 111, 1

Benz, A. O., \& Smith, D. F. 1987, Sol. Phys., 107, 299

Benz, A. O., Csillaghy, A., \& Aschwanden, M. J. 1996, A\&A, 309, 291

Brueckner, G. E., \& Bartoe, J.-D. F. 1983, ApJ, 272, 329

Cairns, I. H. 1990, J. Geophys. Res., 95, 15, 167

Cairns, I. H., \& Gurnett, D. A. 1991a, J. Geophys. Res., 96, 7591

Cairns, I. H., \& Gurnett, D. A. 1991b, J. Geophys. Res., 96, 13,913

Cairns, I. H., \& Zank, G. P. 2001, in Proc. Cospar Colloquium on the Outer Heliosphere: The Next Frontiers, eds K. Scherer, H. Fichtner, E. Marsch, \& H. Fahr, in press

Cranmer, S. R. 2000, ApJ, 532, 1197

Dere, K. P. 1994, Adv. Space Res., 14, 13

Dere, K. P., Bartoe, J.-D. F., \& Brueckner, G. E. 1989, Solar Phys., 123,41

Dennis, B. R. 1985, Sol. Phys., 100, 465

Dulk, G. A., Steinberg, J.-L., Haong, S., \& Goldman, M. V. 1987, A\&A, 173, 366

Fontenla, J. M., Avrett, E. H., \& Loeser, R. 1990, ApJ, 355, 700

Fontenla, J. M., Avrett, E. H., \& Loeser, R. 1991, ApJ, 377, 712

Fontenla, J. M., Avrett, E. H., \& Loeser, R. 1993, ApJ, 406, 319

Gary, S. P. 1993, Theory of Space Plasma Microinstabilities (Cambridge: Cambridge University Press)

Gabriel, A. H. 1971, Sol. Phys., 21, 392

Harrison, R. A. 1997, Sol. Phys., 175, 467

Harrison, R. A., Lang, J., Brooks, D. H., \& Innes, D. E. 1999, A\&A, 351, 1115

Hoang, S., Dulk, G. A., \& Leblanc, Y. 1994, A\&A, 289, 957

Holman, G. D. 1985, ApJ, 293, 584

Innes, D. E., \& Toth, G. 1999, Sol. Phys., 185, 127

Innes, D. E., Inhester, B., Axford, W. I., \& Wilhelm, K. 1997a, Nature, 386, 811
Innes, D. E., Brekke, P., Germott, D., \& Wilhelm, K. 1997b, Sol. Phys., 175, 341

Kohl, J. L., et al., 1997, Sol. Phys., 175, 613

McBride, J. B., Ott, E., Boris, J. P., \& Orens, J. H. 1972, Phys. Fluids, 15,2367

McClements, K. G., Bingham, R., Su, J. J., Dawson, J. M., \& Spicer, D. S. 1993, ApJ, 409, 465

McKenzie, J. F., Banaszkiewicz, M., \& Axford, W. I. 1995, A\&A, 303, L45

McLean, D. J., \& Labrum, N. R. (eds) 1985, Solar Radiophysics (Cambridge: Cambridge University Press)

Melrose, D. B. 1974, Sol. Phys., 37, 353

Melrose, D. B. 1980, Plasma Astrophysics, Vol. II (New York: Gordon \& Breach)

Miller, J. A., et al. 1997, J. Geophys. Res., 102, 14, 631

Omelchenko, Y. A., Sagdeev, R. A., Shapiro, V. D., \& Shevchenko, V. I. 1989, Sov. J. Plasma Phys., 15, 427

Parker, E. N. 1963, ApJS, 8, 177

Parker, E. N. 1990, Geophys. Res. Lett., 17, 2055

Papadopoulos, K. D. 1984, Radio Science, 19, 571

Phan, T., et al. 2000, Nature, 404, 848

Poquerusse, M. 1994, A\&A, 286, 611

Scudder, J. D. 1992, ApJ, 398, 299

Shapiro, V. D., Bingham, R., Dawson, J. M., Dobe, Z., Kellett, B. J., \& Mendis, D. A. 1998, Physica Scr., T75, 39

Shklovskii, I. S. 1965, Physics of the Solar Corona (Oxford: Pergamon)

Spicer, D. S., Benz, A. O., \& Huba, J. D. 1981, A\&A, 105, 221

Tandberg-Hanssen, E., \& Emslie, A. G. 1988, The Physics of Solar Flares (Cambridge: Cambridge University Press)

Wild, J. P. 1950, Aust. J. Sci. Res., A3, 541

Wu, C. S. 1996, ApJ, 472, 818

Wu, C. S., Li, Y., Chao, J. K., Yoon, P. H., \& Lee, L. C. 1998, ApJ, 495, 951

Wang, X. Y., Wu, C. S., Wang, S., Chao, J. K., Lin, Y., \& Yoon, P. H. 2001, ApJ, 547, 1159 